\section{La proctologie en deuil Jean Denis nous a quittés}

\author{
A. Senéjoux \\ (C) Springer-Verlag France 2013
}

C'est avec une immense émotion que j'ai appris le décès de mon maître et ami (Fig. 1).

Si Jean Arnous avait initié le concept de " proctologie à la française » en engageant les gastroentérologues dans de meilleures connaissances et pratiques de la proctologie, Jean Denis l'avait hissé au rang d'école. Travailleur infatigable, il était devenu chef du service de coloproctologie en 1980. Il occupera ce poste pendant plus de 20 ans et fera partager sa passion pour la proctologie médicochirurgicale à de nombreux élèves. Brillant opérateur, il excellait en particulier dans la prise en charge des fistules complexes pour lesquelles il avait une affection toute particulière.

C'était un homme intelligent, à l'humour vif. Je m'en souviendrai comme d'un homme secret : né à Verdun en 1934, une éducation au Prytanée militaire...finalement, il laissait peu transparaître les événements d'une vie privée assez « baroque » ...

Il aimait profondément la vie et ses plaisirs.

Admirer les jolies femmes, mais aussi les enfants jouant dans la cour de la crèche sur laquelle donnaient les fenêtres du couloir de la consultation de proctologie à l'hôpital Léopold Bellan....

Hédoniste, il affectionnait les bonnes tables, discutait de ses recettes de cuisine avec ses aides opératoires et se réjouissait de partager un chardonnay américain et des " shrimps cocktail » un soir de congrès ! J'ai le souvenir mémorable d'un jambalaya de Louisiane commandé, spécialement pour ses amis, au chef d'un bon restaurant Parisien... Ou de ce souvenir d'enfance qu'il aimait me rappeler à table : « Agnès, ma mère me disait, quand j'étais enfant, mon fils, quand le plat de la vie passe, sers toi plutôt deux fois qu'une, tu n'es pas sûr qu'il repassera...».

Cultivé, il aimait la peinture, les récits de marins ou de flibustiers, les biographies mais aussi les polars de gare ou les films de Michel Audiard, se faisant un plaisir de déclamer

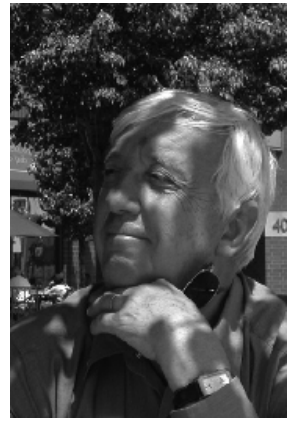

Fig. 1 Jean Denis

les répliques des Tontons Flingueurs en disséquant, le geste rapide et assuré, les quatre paquets hémorroïdaires d'une intervention dite de Bellan...

C'était un grand voyageur, toujours entre deux avions, il adorait tout particulièrement l'Asie, se plaisait à acheter des boîtes birmanes au marché de Chatuchak, souvent pour les offrir à ses amis...ou à évoquer l'aumône matinale des bonzes de Luang Prabang qu'un jour il me fit découvrir...

Il aimait être entouré d'élèves venus de tous les horizons et enseigner l'art de chercher un orifice interne de fistule, et se plaisait à répéter, provocateur, qu'il n'y a pas de fistule borgne mais bien souvent des chirurgiens aveugles. Curieux, les progrès technologiques suscitaient son intérêt, il se plaisait à rapporter dans un compte rendu de congrès telle ou telle nouveauté, souvent pour rester dubitatif... et finalement le temps, et le fil des publications lui donnaient bien souvent raison. Le traitement classique, à la manière de nos anciens maîtres, par fractionnement des temps opératoires et abaissements successifs du trajet demeure en effet une valeur sûre pour le traitement de bien des fistules anales hautes où tout a echoué....Exige beaucoup de toi-même et attends peu des autres. Ainsi beaucoup d'ennuis te seront épargnés. [Confucius]

A. Senéjoux $(\square)$

e-mail : agnes.senejoux@gmail.com 